OPEN ACCESS

Edited by:

Chunfu Zheng,

University of Calgary, Canada

Reviewed by:

Libin Liang,

Shanxi Agricultural University, China

Gang Wang,

Shandong Agricultural University,

China

*Correspondence:

Yulong Gao

gaoyulong@caas.cn

Kai L

likai01@caas.cn

Specialty section:

This article was submitted to

Virology,

a section of the journal

Frontiers in Microbiology

Received: 11 November 2021 Accepted: 06 December 2021 Published: 22 December 2021

Citation:

Yang F, LiU P, Li X, LiU R, Gao L, Cui $H$, Zhang Y, Liu C, Qi X, Pan Q, Liu A, Wang X, Gao Y and Li K (2021)

Recombinant Duck Enteritis Virus-Vectored Bivalent Vaccine Effectively Protects Against Duck

Hepatitis A Virus Infection in Ducks.

Front. Microbiol. 12:813010

doi: 10.3389/fmicb.2021.813010

\section{Recombinant Duck Enteritis Virus-Vectored Bivalent Vaccine Effectively Protects Against Duck Hepatitis A Virus Infection in Ducks}

\author{
Fuchun Yang, Peng Liu, Xiaohan Li, Rui Liu, Li Gao, Hongyu Cui, Yanping Zhang, \\ Changjun Liu, Xiaole Qi, Qing Pan, Aijing Liu, Xiaomei Wang, Yulong Gao* and Kai Li*
}

Avian Immunosuppressive Diseases Division, State Key Laboratory of Veterinary Biotechnology, Harbin Veterinary Research Institute, Chinese Academy of Agricultural Sciences, Harbin, China

Duck enteritis virus (DEV) and duck hepatitis A virus (DHAV) are prevalent duck pathogens, causing significant economic losses in the duck industry annually. Using a fosmid-based rescue system, we generated two DEV recombinants, rDEV-UL26/27P13C and rDEV-US7/8-P13C, in which the P1 and 3C genes from DHAV type 3 (DHAV-3) were inserted into the DEV genome between genes UL26 and UL27 or genes US7 and US8. We inserted a self-cleaving 2A-element between P1 and 3C, allowing the production of both proteins from a single open reading frame. $\mathrm{P} 1$ and $3 \mathrm{C}$ were simultaneously expressed in infected chicken embryo fibroblasts, with no difference in growth kinetics between cells infected with the recombinant viruses and those infected with the parent DEV. Both recombinant viruses induced neutralizing antibodies against DHAV-3 and DEV in ducks. A single dose of the recombinant viruses induced solid protection against lethal DEV challenge and completely prevented DHAV-3 infection as early as 7 days post-vaccination. These recombinant P1- and 3C-expressing DEVs provide potential bivalent vaccines against DEV and DHAV-3 infection in ducks.

Keywords: duck enteritis virus, duck hepatitis $\mathrm{A}$ virus, bivalent vaccine, $\mathrm{P} 1$ protein, $3 \mathrm{C}$ protein

\section{INTRODUCTION}

Ducks have many commercial uses, making them one of the most important waterfowl. However, duck production is threatened by many pathogens, of which the most important are duck hepatitis A virus (DHAV) and duck enteritis virus (DEV, a herpesvirus) (Yugo et al., 2016; Dhama et al., 2017). This is a major factor in China, where up to four billion ducks are reared annually for food and clothing (Liu et al., 2011a).

Duck hepatitis A virus belongs to the genus Avihepatovirus in the family Picornaviridae. It has a linear, single-stranded positive-sense RNA genome comprising a large open reading frame encoding a polyprotein, as well as untranslated regions (Wen et al., 2018). Its P1 region encodes the $\mathrm{P} 1$ protein, which is translated within this large open reading frame and is hydrolyzed into the structural capsid proteins VP0, VP1, and VP3 by the viral 3C protease (Kim et al., 2006; Wang et al., 2018). DHAV is the most common cause of duck virus hepatitis (DVH), which induces an acute, highly contagious, and rapidly progressing fatal disease in young ducklings, causing substantial economic losses in the duck industry worldwide (Wen et al., 2018). The DHAV strains are classified 
into three serotypes: the traditional serotype 1 (DHAV-1), distributed worldwide (Wang et al., 2008); serotype 2 (DHAV-2), endemic only in Taiwan (Tseng and Tsai, 2007); and the novel serotype 3 (DHAV-3). In Southeast Asia, DVH is caused mainly by DHAV-1 and DHAV-3 (Wen et al., 2018). DHAV-1 infection is currently controlled using modified live attenuated vaccines (Roh and Kang, 2018); in China, this has been officially approved for vaccinating breeder ducks since 2013. Although DHAV-3 accounts for an increasing proportion of DHAV infections in mainland China (Liu et al., 2011b), South Korea (Kim et al., 2007), and Vietnam (Roan et al., 2015), no commercial live vaccine suitable used in young ducklings is currently available. A safe and efficient vaccine for DHAV-3 is required.

Duck enteritis virus, also called duck plague virus, causes an acute contagious disease among Anseriformes (ducks, geese, and swans), resulting in high mortality and reduced egg production in domestic and wild waterfowl (Dhama et al., 2017). Attenuated DEV strains, including C-KCE and clone 03 from embryonated chicken eggs, have been routinely used as live vaccines in ducks for over half a century, without posing safety concerns for humans and domestic animals (Liu et al., 2011a; Sun et al., 2017). As with other herpesviruses, the large genome of DEV (approximately $158 \mathrm{~kb}$ ) makes it highly suitable as a live viral vector for inserting and expressing the foreign antigen genes of other pathogens and for developing multivalent vaccines (Liu et al., 2011a; Chen et al., 2014; Zou et al., 2016; Sun et al., 2017).

Mixed DHAV and DEV infection has increasingly become frequent in domestic ducks, worsening the economic loss. Developing a bivalent vaccine that simultaneously acts against DHAV-3 and DEV is the most economical method for reducing losses in the duck industry. To address this, we generated two recombinant DEVs, with the $\mathrm{P} 1$ and $3 \mathrm{C}$ genes of a DHAV-3 virus inserted at different sites in the $\mathrm{DEV}$ vaccine strain genome via transfection of overlapping fosmid DNAs. The recombinant viruses were further evaluated in vitro and in vivo for antigen expression, replication, stability, and protective efficacy against DHAV-3 and DEV challenge in ducks.

\section{MATERIALS AND METHODS}

\section{Animals and Ethics Statement}

The specific-pathogen-free (SPF) ducks, fertilized SPF chicken eggs, and duck eggs used in this study were purchased from the State Resource Center of Laboratory Animal for Poultry (Harbin, China). Ten-day-old SPF chicken and duck embryos were used to prepare primary chicken embryo fibroblasts (CEFs) and duck embryo fibroblasts (DEFs). This study was carried out according to the Guide for the Care and Use of Laboratory Animals of the Ministry of Science and Technology of China. The use of SPF eggs, embryos, and ducks and the animal experiments were approved by the Animal Ethics Committee of Harbin Veterinary Research Institute of the Chinese Academy of Agricultural Sciences and performed following animal ethics guidelines and approved protocols [SYXK (Hei) 2017009].

\section{Viruses and Cells}

The attenuated DEV vaccine strain C-KCE (Yang et al., 2013) was propagated in primary CEFs and used as the parent virus to produce the recombinant DEV. The virulent DEV CSC (Yang et al., 2014) and DHAV-3 A3 strains were propagated in 10-dayold SPF duck embryonated eggs and used as the challenge viruses.

\section{Construction of Fosmids With Insertion of DHAV-3 P1 and 3C Genes}

Five fosmids (C027, C018, C144, C211, and C343), containing sequences spanning the entire genome of the $\mathrm{DEV}$ vaccine strain C-KCE (GenBank accession no. KF263690) were constructed during our preliminary studies and used for insertion of DHAV3 P1 and 3C genes (Figure 1A). A dual-selection marker cassette containing the kanamycin resistance gene (KanR) and the ccdB gene flanked by the attR1 and attR2 sequences was first inserted into the gene junction between the UL26 and UL27 genes, or between the US7 and US8 genes, in the C144 or C343 fosmids, via Red/ET recombination using the counter-selection BAC modification kit (Gene Bridges $\mathrm{GmbH}$, Heidelberg, Germany) to facilitate the insertion of foreign genes into the DEV genome. The modified fosmids containing the selection markers were designated C144-UL26/27-KanccdB and C343-US7/8-KanccdB, respectively.

The gene fragment $\mathrm{P} 1-\mathrm{P} 2 \mathrm{~A}-3 \mathrm{C}$, containing the $\mathrm{P} 1$ and $3 \mathrm{C}$ genes of DHAV-3 and a self-cleaving $2 \mathrm{~A}$-element of porcine teschovirus-1 (Szymczak-Workman et al., 2012), was synthesized and cloned into the pCAGGS vector under the control of the CMV enhancer and $\beta$-actin promoter. The constructed P1-P2A-3C cassette was then used to replace the gus gene in the plasmid pENTR-gus (Invitrogen), and the resultant entry plasmid was designated pENTRP13C. To insert the P1-P2A-3C cassette into the DEV genome, the entry plasmid pENTR-P13C was mixed with the modified fosmids C144-UL26/27-KanccdB and C343-US7/8-KanccdB and treated with LR Clonase II enzyme (Invitrogen). The mixtures were transformed into competent Escherichia coli EPI300-T1 cells. The resultant fosmids with P1-P2A-3C cassette insertion were designated C144-UL26/27-P13C (Figure 1B) and C343-US7/8-P13C (Figure 1C).

\section{Rescue of Recombinant Viruses From Overlapping Fosmid DNAs}

The five fosmids with or without P1-P2A-3C insertion were used to rescue the recombinant viruses. Viral DNA inserts were released from the purified fosmids by digestion with SbfI and were purified via phenol-chloroform extraction and ethanol precipitation before transfection. Two micrograms of each fosmid DNA were used to transfect the primary CEFs in $60 \mathrm{~mm}$ dishes, using the calcium phosphate procedure (Morgan et al., 1990). The cytopathic effect-positive samples were harvested and characterized via electron microscopy. To verify that the P1P2A-3C cassette was inserted at the desired sites, viral genomic DNA was isolated from the rescued viruses and analyzed using PCR and sequencing. 


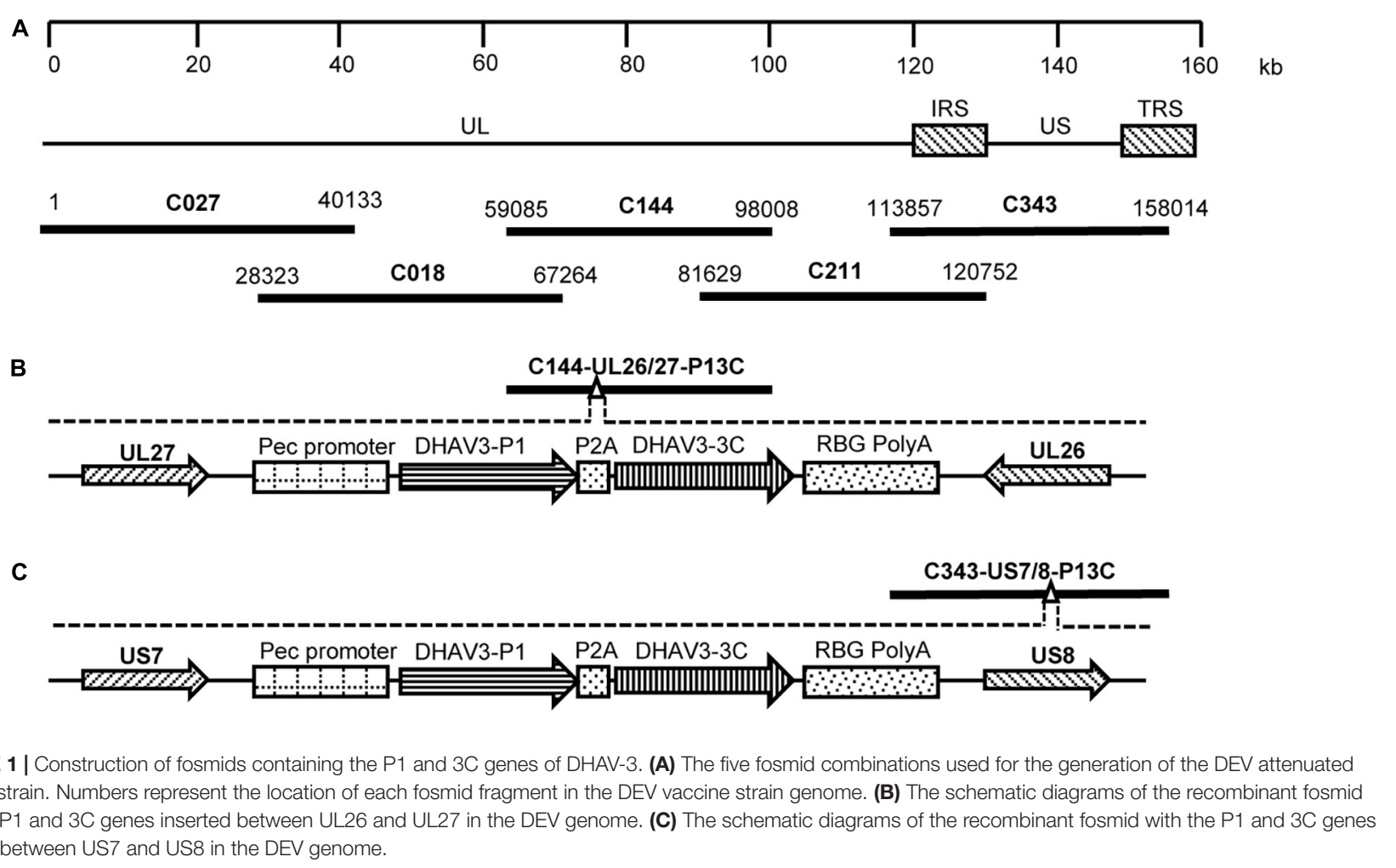

\section{Confirmation of P1 and 3C Gene Expression}

Expression of DHAV-3 P1 and 3C by recombinant DEVs was confirmed via indirect immunofluorescence assay. Briefly, CEFs in 6-well tissue culture plates were infected with the rescued viruses for 3 days. The medium was aspirated, and the cells were fixed with absolute ethanol for $20 \mathrm{~min}$ at room temperature. The fixed cells were incubated with rabbit anti-DHAV3-VP1 or rabbit anti-DHAV3-3C polyclonal antibodies in a wet box for $60 \mathrm{~min}$ at $37^{\circ} \mathrm{C}$, then reacted with TRITC-labeled goat anti-rabbit IgG antibody (1:100 solution) (Sigma, St. Louis, MO, United States) in a wet box for $60 \mathrm{~min}$ at $37^{\circ} \mathrm{C}$. After being washed five times, the cells were examined via fluorescence microscopy.

\section{Growth and Stability of the Recombinant Viruses}

Chicken embryo fibroblasts cultured in 6-well plates were inoculated with different viruses at a multiplicity of infection of 0.01 and maintained at $37^{\circ} \mathrm{C}$ in $5 \% \mathrm{CO}_{2}$ for 4 days to investigate the growth of the recombinant DEVs. The cells and supernatants were harvested at $24 \mathrm{~h}$ intervals, and the titer of infectious progeny present in the culture was determined as the median tissue culture infectious dose $\left(\mathrm{TCID}_{50}\right)$ per $\mathrm{mL}$, using the Reed-Muench formula (Reed and Muench, 1938). The mean \pm standard deviation (SD) was calculated from three independent experiments. To evaluate the genetic stability of the foreign gene in the recombinant virus, we passaged the virus in the CEFs 20 times. The inserted genes were detected via PCR and sequencing, and $\mathrm{P} 1$ and $3 \mathrm{C}$ expression were confirmed using immunofluorescence assay as described.

\section{Protection Against DHAV-3 and Duck Enteritis Virus Challenge}

All the animal experiments were performed using SPF ducks housed in filtered-air, negative-pressure isolation units. The ducks were given free access to food and water. To evaluate the protective efficacy of the recombinant viruses against challenge by the virulent DHAV-3 and DEV, each group of 20 ducks was inoculated subcutaneously with 1,000 times the 50\% egg lethal dose $\left(1000 \mathrm{ELD}_{50}\right)$ of the rescued viruses at 1 day of age. At 7 days post-vaccination, 10 ducks in each group were intramuscularly challenged with 100 ELD $_{50}$ of the virulent DHAV-3 A3 strain, and the remaining 10 ducks were intramuscularly challenged with 1,000 minimum lethal doses of the virulent DEV CSC strain. Ten unvaccinated and unchallenged ducks were used as the healthy controls. The ducks were examined for clinical signs and mortality for 2 weeks after the challenge. The dead and surviving ducks were observed for gross lesions in the liver, spleen, kidneys, esophagus, intestine, thymus, and bursa.

\section{Serological Tests}

Serum samples were collected from ducks 7 days post-vaccination and tested via virus neutralization assays. To determine the viral neutralizing antibody against DHAV-3, triplicates of heatinactivated serum samples were serially diluted two-fold and mixed with an equal volume of $100 \mathrm{ELD}_{50}$ of the virulent DHAV-3 A3 strain. After $1 \mathrm{~h}$ of incubation, the mixtures were 
added to 10-day-old embryonated SPF duck eggs, followed by further incubation for 7 days. The neutralizing antibody titer was determined as $\log _{2}$ of the reciprocal of the highest dilution at which there were no dead eggs.

To determine the viral neutralizing antibody against DEV, triplicates of heat-inactivated serum samples were serially diluted two-fold and mixed with an equal volume of 100 TCID $_{50}$ of the virulent DEV CSC strain. After $1 \mathrm{~h}$ of incubation, the mixtures were added to monolayers of DEFs, followed by further incubation for 5 days. The neutralizing antibody titer was determined as $\log _{2}$ of the reciprocal of the highest dilution at which there were no visible cytopathic effects.

\section{Statistical Analysis}

The results are presented as mean \pm SD. One-way ANOVA was used to evaluate the statistical difference among groups, using SPSS 17.0 (SPSS Inc., Chicago, IL, United States). Statistical significance was set at $P<0.05$ for all tests.

\section{RESULTS}

\section{Generation of Recombinant Duck Enteritis Viruses Expressing DHAV-3 P1 and $3 \mathrm{C}$ Genes}

The P1-P2A-3C cassette was inserted into the gene junction between UL26 and UL27, or between US7 and US8, in the DEV vaccine strain genome. For transfection, we used the modified fosmids, C144-UL26/27-P13C (Figure 1B) and C343-US7/8P13C (Figure 1C), which replaced the parental fosmids, C144 and $\mathrm{C} 343$, respectively. After being blindly passaged once in CEFs, DEV-typical plaques appeared in the CEFs transfected with the DNA combinations (Figure 2A). Electron microscopy confirmed the successful rescue of the recombinant viruses (Figure 2B). Insertion of the P1-P2A-3C cassette at the proper site was confirmed by PCR and sequencing, using a forward primer specific to the $\mathrm{P} 1$ gene and a reverse primer matching the UL26 or US8 sequences (Figure 3A). The recombinant DEVs were designated rDEV-UL26/27-P13C, with the P1 and 3C genes inserted between UL26 and UL27, and rDEV-US7/8P13C, with the P1 and 3C genes inserted between US7 and US8, in the DEV genome.

The expression of $\mathrm{P} 1$ and $3 \mathrm{C}$ proteins by the recombinant viruses was confirmed via indirect immunofluorescence assay. The cells infected with rDEV-UL26/27-P13C and rDEV-US7/8$\mathrm{P} 13 \mathrm{C}$ reacted with both the anti-DHAV3-VP1 and anti-DHAV3$3 \mathrm{C}$ antibodies, emitting a red fluorescent signal, whereas the parental virus-infected cells did not react with the antibodies (Figure 3B). These results indicate that recombinant DEVs coexpressing DHAV-3 P1 and 3C genes were successfully generated.

\section{Growth Kinetics and Genetic Stability of the Recombinant Duck Enteritis Viruses}

We tested whether insertion of the P1-P2A-3C cassette affected the in vitro replication of DEV. The CEF cultures infected with the viruses were harvested at different time points for titration in 96-well plates. The in vitro growth kinetics and magnitude of the two recombinant DEVs expressing DHAV-3 P1 and 3C were very similar to the parental virus rDEV-WT (Figure 4A), indicating that insertion of $\mathrm{P} 1$ and $3 \mathrm{C}$ did not affect the replication of the DEV vaccine strain.

To investigate whether the inserted $\mathrm{P} 1$ and $3 \mathrm{C}$ genes could be stably maintained in the recombinant viruses, we passaged the viruses in CEFs 20 times. The P1-P2A-3C cassette in both recombinants was detected by PCR amplification (Figure 4B). P1 and 3C protein expression by the serially passaged viruses was confirmed via immunofluorescence after 20 passages (Figure 4C).

\section{Antibody Responses Against DHAV-3 and Duck Enteritis Virus Induced by Recombinant Duck Enteritis Viruses in Ducks}

Groups of 20 ducks were inoculated with $1000 \mathrm{ELD}_{50}$ of the recombinant viruses to evaluate the antibody responses against DHAV-3 and DEV induced by the recombinant DEVs. Anti-DHAV-3 neutralizing antibodies were detected from all ducks immunized with rDEV-UL26/27-P13C or rDEV-US7/8$\mathrm{P} 13 \mathrm{C}$, as early as 7 days post-inoculation with mean titers of 7.3 and 6.7 ( $\log _{2}$ values), respectively. The anti-DHAV3 antibodies induced by these two viruses were comparable, with no significant difference $(P>0.05)$ (Figure 5A). The recombinant viruses rDEV-UL26/27-P13C and rDEV-US7/8$\mathrm{P} 13 \mathrm{C}$ also induced measurable anti-DEV neutralizing antibodies at 7 days post-inoculation, with mean titers of 3.7 and $2.6\left(\log _{2}\right.$ values), respectively. The recombinant virus rDEV-UL26/27$\mathrm{P} 13 \mathrm{C}$ produced a higher level of neutralizing antibodies against DEV than rDEV-US7/8-P13C $(p<0.05)$ (Figure 5B).

\section{Protective Efficacy Against Lethal DHAV-3 and Duck Enteritis Virus Challenge in Ducks}

The ducks were inoculated with the recombinant viruses at 1 day of age and challenged with the virulent DHAV-3 A3 strain 7 days post-inoculation for protective efficacy evaluation. These ducks did not show any clinical signs in response to vaccination before the challenge. As shown in Figure 6A, the ducks in the rDEVUL26/27-P13C and rDEV-US7/8-P13C vaccination groups were completely protected from lethal DHAV-3 challenge, showing no clinical signs of disease and no visible lesions in the liver, spleen, or other organs during the 2 -week observation period. However, all ducks in the challenge control group showed severe clinical signs from 2 days post-challenge, including depression, lethargy, and anorexia; all these ducks died from the DHAV-3 challenge within 3 days. As expected, the ducks in the healthy control group did not show any clinical signs during the experiment. These results indicate that rDEV-UL26/27-P13C and rDEVUS7/8-P13C induced $100 \%$ protection against lethal DHAV-3 challenge in ducks.

We then evaluated the protective efficacy of the recombinant viruses against the DEV challenge. After the challenge, all the ducks in the challenge control group showed signs of disease, 

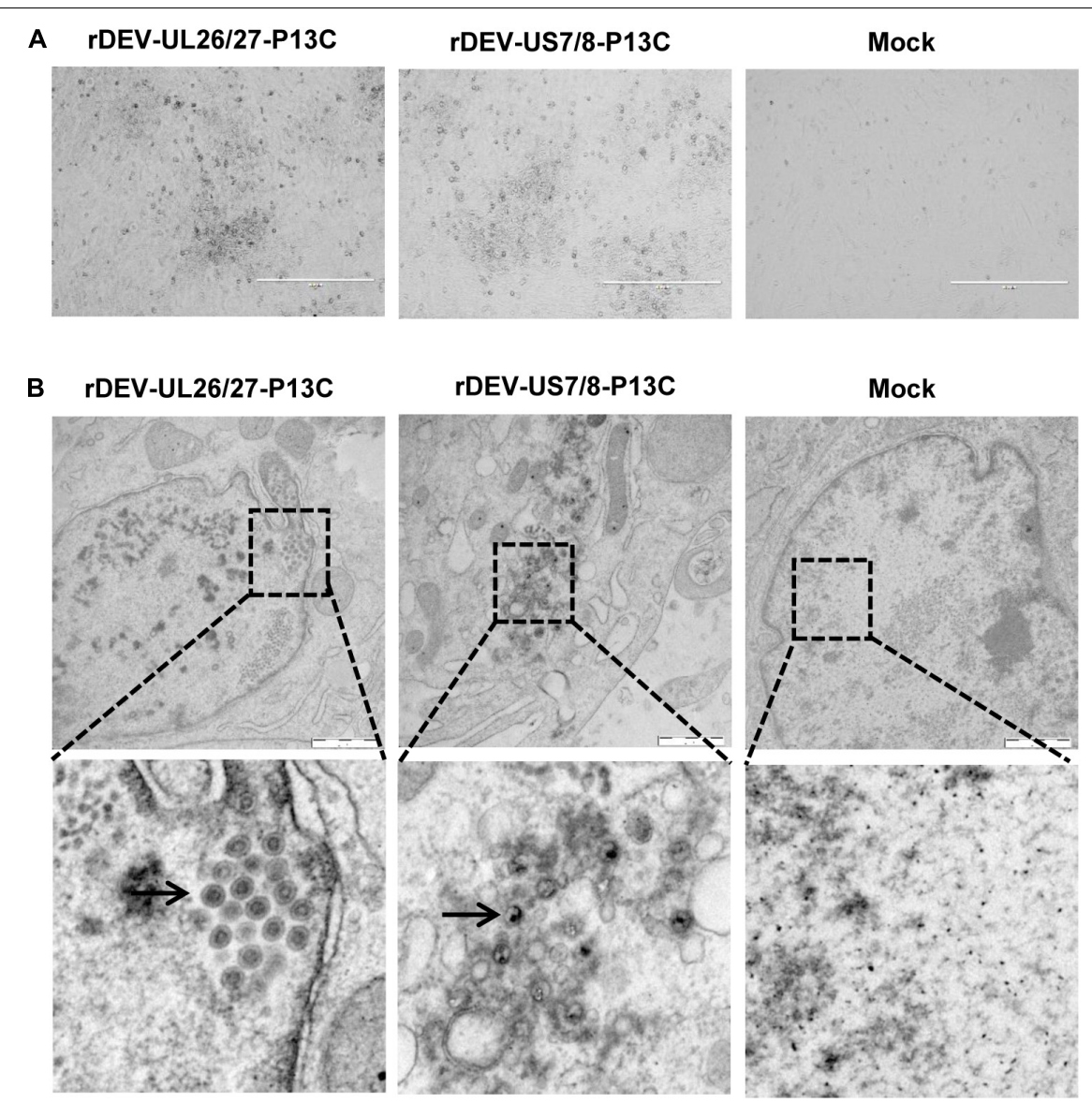

FIGURE 2 | Generation of recombinant DEVs with the P1 and 3C genes insertion. (A) The cytopathic effects induced by the recombinant DEVs containing the DHAV-3 P1 and 3C genes in CEFs. (B) Electron microscopy detection of the recombinant DEVs in CEFs. Bar length, $1 \mu \mathrm{m}$. Arrows represent the DEV virions detected in infected cells.

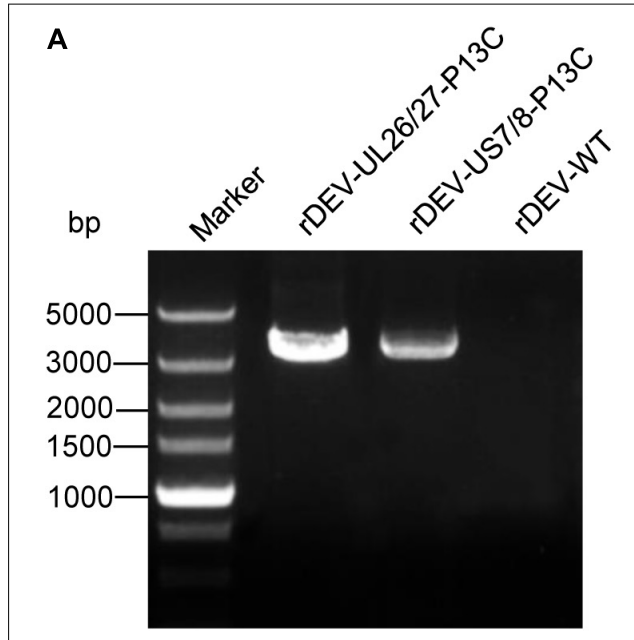

B

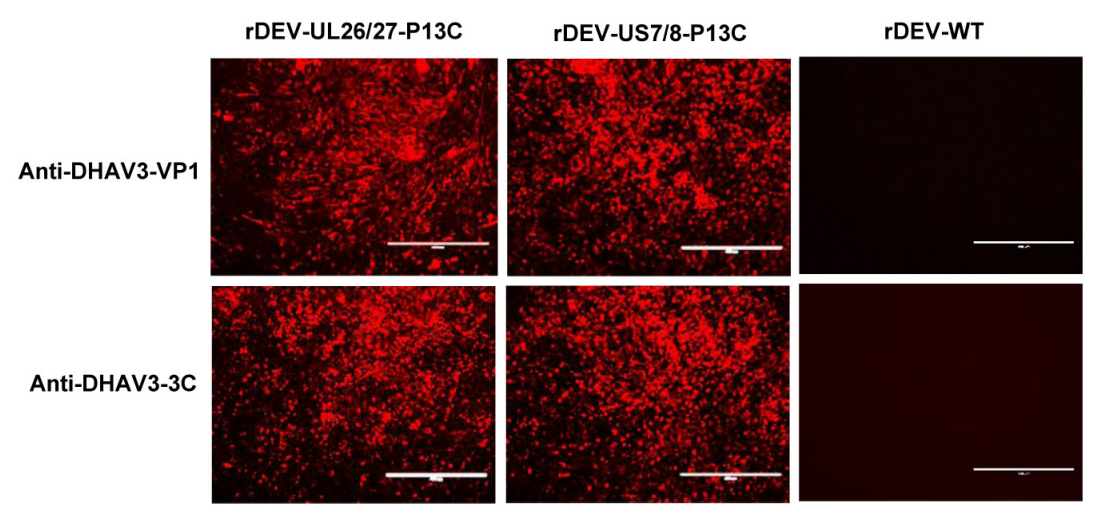

FIGURE 3 | Detection of P1 and 3C expression by recombinant DEVs in infected CEFs. (A) PCR amplification of the P1-P2A-3C cassette from the recombinant viruses. (B) Detection of P1 and $3 \mathrm{C}$ expression by the recombinant viruses in indirect immunofluorescence assay with anti-DHAV3-VP1 and anti-DHAV3-3C antibodies. 
A

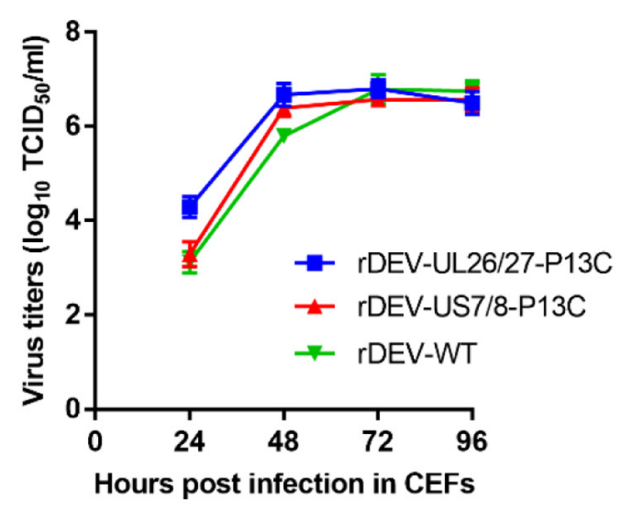

C

Anti-DHAV3-VP1

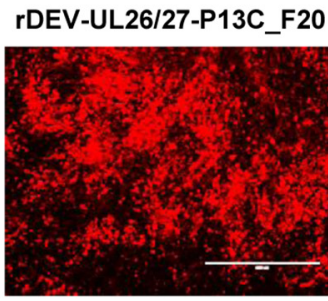

Anti-DHAV3-3C

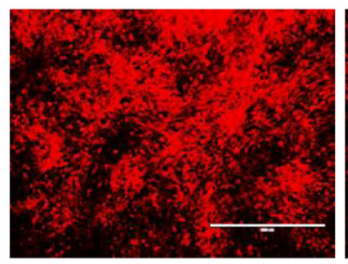

B

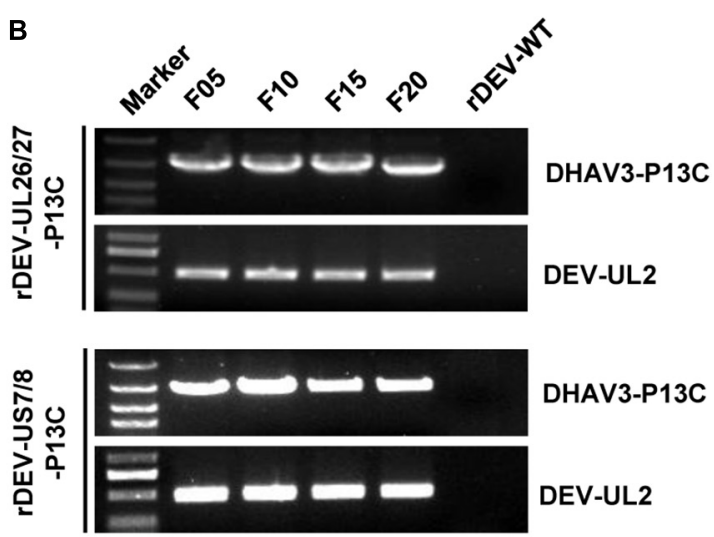

rDEV-WT
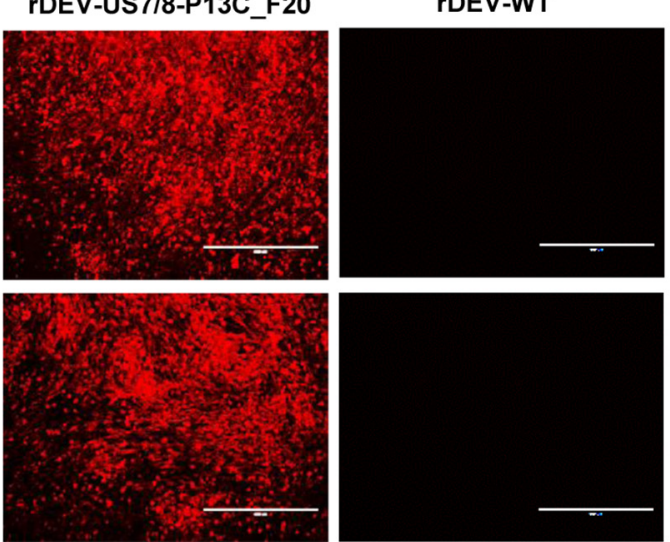

FIGURE 4 | Growth kinetics and genetic stability of the recombinant DEVs in CEFs. (A) Comparison of the replication kinetics of the recombinant DEVs and the parental virus (rDEV-WT) in CEFs. (B) Detection of the P1 and 3C genes inserted in the recombinant DEVs passaged 5 (F05), 10 (F10), 15 (F15), and 20 (F20) times in CEFs. (C) Confirmation of P1 and 3C expression by the recombinant DEVs passaged 20 times in CEFs with immunofluorescence assay.
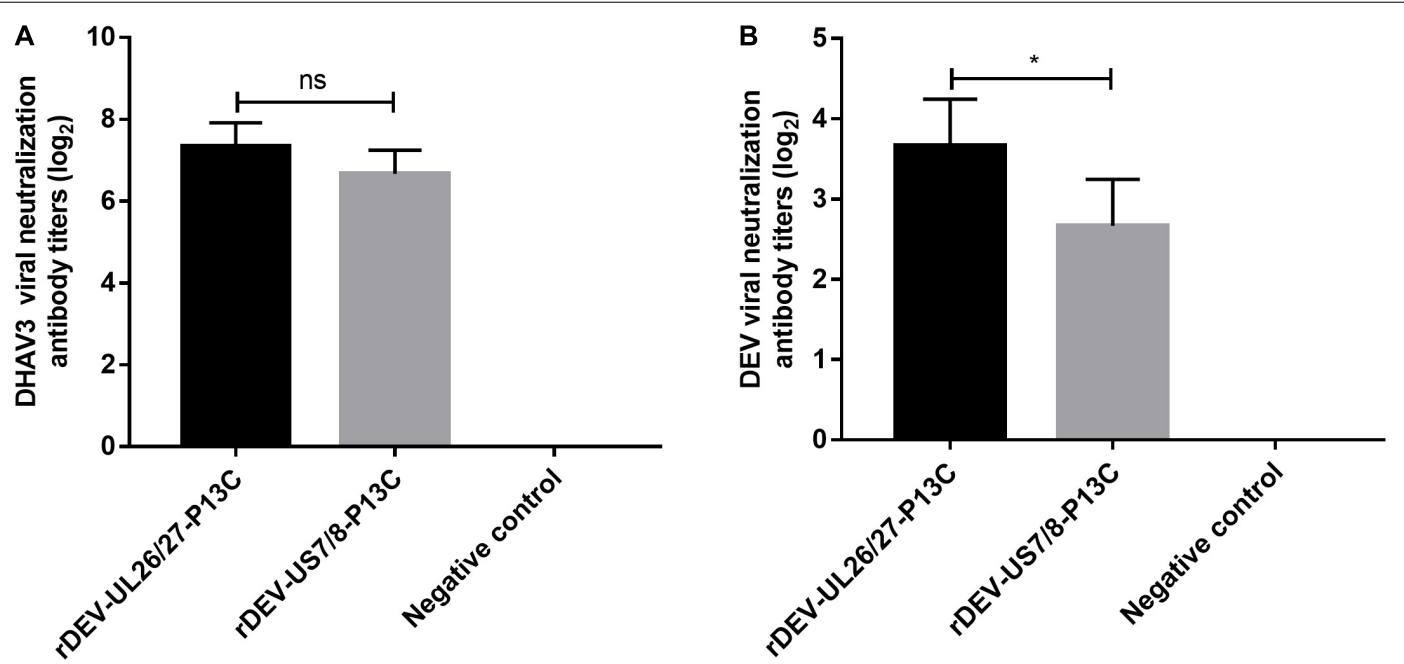

FIGURE 5 | Antibody responses induced by the recombinant DEVs in ducks. Groups of twenty ducks were inoculated with 1000 ELD 50 of the recombinant DEVs, and the sera samples were collected after 7 days of vaccination to detect neutralizing antibodies against DHAV-3 (A) and DEV (B). The neutralizing antibody titers for ducks are expressed as $\log _{2}$. Data presented are the means \pm standard deviations from twenty ducks per group. ns, no significant difference; ${ }^{*} P<0.05$. 

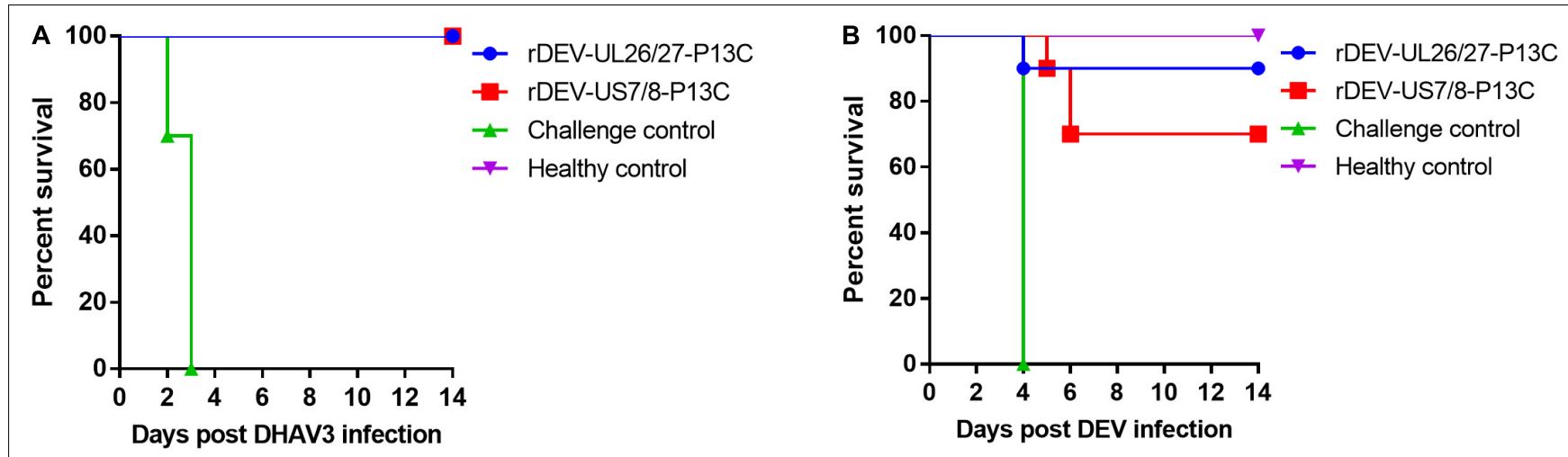

FIGURE 6 | Protective efficacy of the recombinant DEVs against lethal DHAV-3 and DEV challenge in ducks. Ducks were inoculated with 1000 ELD 50 of the recombinant DEVs and then challenged with 100 ELD $_{50}$ of the virulent DHAV-3 A3 strain or 1000 minimum lethal doses of the virulent DEV CSC strain after 7 days of vaccination. (A) The survival rate of ducks challenged with DHAV-3 within an observation period of 14 days. (B) The survival rate of ducks challenged with DEV within an observation period of 14 days.

including listlessness, ruffled feathers, and anorexia, and died at 4 days post-challenge. Ninety percent $(9 / 10)$ of the ducks in the rDEV-UL26/27-P13C group survived the DEV challenge and showed no clinical signs or gross lesions, indicating that rDEVUL26/27-P13C vaccination provided $90 \%$ protection against DEV infection (Figure 6B). In comparison, vaccination with rDEV-US7/8-P13C conferred 70\% (7/10) protection against DEV challenge. These results suggest that the recombinant viruses also induced solid protection against lethal DEV challenge in ducks.

\section{DISCUSSION}

Duck hepatitis A virus and DEV are highly prevalent pathogens in duck farms worldwide, resulting in more than $80 \%$ of mortality in ducklings (Chen et al., 2013; Li et al., 2013; Soliman et al., 2015; Lin et al., 2016). Vaccination provides the most effective protection against DHAV and DEV infection in ducks (Yugo et al., 2016; Dhama et al., 2017). The prevention of DVH mainly depends on the immunization of ducklings with commercial DHAV-1 live vaccines. However, the DHAV-1 vaccine failed to elicit cross-protection against DHAV-3, which currently severely affects Southeast Asia (Kim et al., 2009; Roan et al., 2015). Furthermore, simultaneous co-infection with DHAV and DEV has become increasingly prevalent in domestic duck farms in China and South Korea (Chen et al., 2013; Soliman et al., 2015). Thus, an effective and convenient bivalent vaccine against both DHAV-3 and DEV is highly recommended.

Live viral vector vaccines have been proven to effectively control avian diseases (Vannucc et al., 2013). Some recombinant vector vaccines have been granted licenses (Ge et al., 2007; DiNapoli et al., 2010). As a herpesvirus family member, DEV is a highly desirable live virus vector to generate multivalent vaccines. DEV-attenuated live vaccines rapidly induce protective immunity within several hours, and their efficacy appears to be unaffected by maternal antibodies (Liu et al., 2011a). DEV can establish latency after infection and induce long-term humoral and cellular immune responses (Dhama et al., 2017). The natural host range of DEV is limited to the order Anseriformes; hence, as a vaccine vector, it does not pose a risk to other domestic animals or humans. DEV has been widely applied to deliver protective antigens of avian pathogens, including avian influenza virus (Liu et al., 2011a; Sun et al., 2017), duck tembusu virus (Chen et al., 2014; Zou et al., 2016), and Newcastle disease virus (Ding et al., 2019). To address the urgent need for a single bivalent vaccine against both DEV and DHAV-3 infection, we generated two recombinant DEVs, with the P1 and 3C genes of a DHAV-3 virus inserted into the $\mathrm{DEV}$ vaccine strain genome.

To simultaneously express the three capsid proteins of DHAV3 (VP0, VP1, and VP3), we cloned the P1 and 3C genes of DHAV-3 into a single expression vector and separated these genes using a self-cleaving $2 \mathrm{~A}$-element from porcine teschovirus1 (Szymczak-Workman et al., 2012). Our immunofluorescence assay revealed that both $\mathrm{P} 1$ and $3 \mathrm{C}$ were successfully expressed in the cells infected with the recombinant virus. Since duck hepatitis is typically more lethal in younger ducklings, early vaccination of ducklings at hatching is important for preventing DHAV infection. In the present study, we evaluated the protective efficacy of recombinant DEVs against virulent DHAV-3 challenge in 1-day-old SPF ducks. After single-dose immunization, both rDEV-UL26/27-P13C and rDEV-US7/8-P13C elicited rapid immune responses against DHAV-3, as early as 7 days postvaccination, conferring complete protection against DHAV-3 challenge. Moreover, the recombinant DEVs expressing DHAV-3 antigens provided solid protection against lethal DEV challenges. It appears that rDEV-UL26/27-P13C provided better protection against DEV challenge than $\mathrm{rDEV}-\mathrm{US} 7 / 8-\mathrm{P} 13 \mathrm{C}$, suggesting that the UL26-UL27 gene junction site may be better than the US7US8 site for use in developing DEV vector vaccines.

In summary, we developed recombinant DEV vector vaccines expressing the DHAV-3 P1 and 3C genes. These vaccines demonstrated good immunogenicity and provided rapid protection against lethal DHAV-3 and DEV challenges in ducks. The recombinant DEV-vectored DHAV-3 vaccine, which provides effective protection, holds great promise for use as a bivalent vaccine to combat DHAV-3 and DEV infection in ducks. 


\section{DATA AVAILABILITY STATEMENT}

The datasets presented in this study can be found in online repositories. The names of the repository/repositories and accession number(s) can be found at: NCBI GenBank KF263690.

\section{ETHICS STATEMENT}

The animal study was reviewed and approved by the Animal Ethics Committee of Harbin Veterinary Research Institute of the Chinese Academy of Agricultural Sciences and performed in accordance with animal ethics guidelines and approved protocols [SYXK (Hei) 2017-009].

\section{REFERENCES}

Chen, L. L., Xu, Q., Zhang, R. H., Yang, L., Li, J. X., Xie, Z. J., et al. (2013). Improved duplex RT-PCR assay for differential diagnosis of mixed infection of duck hepatitis A virus type 1 and type 3 in ducklings. J. Virol. Methods 192, 12-17. doi: 10.1016/j.jviromet.2013. 04.012

Chen, P., Liu, J., Jiang, Y., Zhao, Y., Li, Q., Wu, L., et al. (2014). The vaccine efficacy of recombinant duck enteritis virus expressing secreted $\mathrm{E}$ with or without PrM proteins of duck tembusu virus. Vaccine 32, 5271-5277. doi: 10.1016/j.vaccine. 2014.07.082

Dhama, K., Kumar, N., Saminathan, M., Tiwari, R., Karthik, K., Kumar, M. A., et al. (2017). Duck virus enteritis (duck plague) - a comprehensive update. Vet. Q. 1, 57-80. doi: 10.1080/01652176.2017.1298885

DiNapoli, J. M., Nayak, B., Yang, L., Finneyfrock, B. W., Cook, A., Andersen, H., et al. (2010). Newcastle disease virus-vectored vaccines expressing the hemagglutinin or neuraminidase protein of $\mathrm{H} 5 \mathrm{~N} 1$ highly pathogenic avian influenza virus protect against virus challenge in monkeys. J. Virol. 84, 14891503. doi: 10.1128/JVI.01946-09

Ding, L., Chen, P., Bao, X., Li, A., Jiang, Y., Hu, Y., et al. (2019). Recombinant duck enteritis viruses expressing the Newcastle disease virus (NDV) F gene protects chickens from lethal NDV challenge. Vet. Microbiol. 232, 146-150. doi: 10.1016/j.vetmic.2019.04.022

Ge, J., Deng, G., Wen, Z., Tian, G., Wang, Y., Shi, J., et al. (2007). Newcastle disease virus-based live attenuated vaccine completely protects chickens and mice from lethal challenge of homologous and heterologous $\mathrm{H} 5 \mathrm{~N} 1$ avian influenza viruses. J. Virol. 81, 150-158. doi: 10.1128/JVI.01514-06

Kim, M. C., Kim, M. J., Kwon, Y. K., Lindberg, A. M., Joh, S. J., Kwon, H. M., et al. (2009). Development of duck hepatitis A virus type 3 vaccine and its use to protect ducklings against infections. Vaccine 27, 6688-6694. doi: 10.1016/j. vaccine.2009.08.092

Kim, M. C., Kwon, Y. K., Joh, S. J., Kim, S. J., Tolf, C., Kim, J. H., et al. (2007). Recent Korean isolates of duck hepatitis virus reveal the presence of a new geno- and serotype when compared to duck hepatitis virus type 1 type strains. Arch. Virol. 152, 2059-2072. doi: 10.1007/s00705-0071023-0

Kim, M. C., Kwon, Y. K., Joh, S. J., Lindberg, A. M., Kwon, J. H., Kim, J. H., et al. (2006). Molecular analysis of duck hepatitis virus type 1 reveals a novel lineage close to the genus Parechovirus in the family Picornaviridae. J. Gen. Virol. 87, 3307-3316. doi: 10.1099/vir.0.81804-0

Li, J., Bi, Y., Chen, C., Yang, L., Ding, C., and Liu, W. (2013). Genetic characterization of duck hepatitis A viruses isolated in China. Virus Res. 178, 211-216. doi: 10.1016/j.virusres.2013.10.007

Lin, S. L., Cong, R. C., Zhang, R. H., Chen, J. H., Xia, L. L., Xie, Z. J., et al. (2016). Circulation and in vivo distribution of duck hepatitis A virus types 1 and 3 in infected ducklings. Arch. Virol. 161, 405-416. doi: 10.1007/s00705-0152648-z

\section{AUTHOR CONTRIBUTIONS}

FY, KL, and YG conceived and designed the experiments. FY and KL performed the experiments and wrote the manuscript. PL, XL, RL, KL, YG, and LG analyzed the data. HC, YZ, CL, XQ, QP, AL, and XW contributed reagents, materials, and analysis tools. All authors read and approved the final manuscript.

\section{FUNDING}

This work was supported by grants from the Heilongjiang Natural Science Foundation of China (YQ2020C024), National Natural Science Foundation of China (31970162 and U20A2061), and China Agricultural Research System (CARS-41-G15).

Liu, J., Chen, P., Jiang, Y., Wu, L., Zeng, X., Tian, G., et al. (2011a). A duck enteritis virus-vectored bivalent live vaccine provides fast and complete protection against H5N1 avian influenza virus infection in ducks. J. Virol. 85, 10989-10998. doi: 10.1128/JVI.05420-11

Liu, M., Meng, F., Li, X. J., Zhang, Z., Liu, S., and Zhang, Y. (2011b). Goose haemorrhagic hepatitis caused by a new subtype duck hepatitis type 1 virus. Vet. Microbiol. 152, 280-283. doi: 10.1016/j.vetmic.2011. 05.015

Morgan, R. W., Cantello, J. L., and McDermott, C. H. (1990). Transfection of chicken embryo fibroblasts with Marek's disease virus DNA. Avian Dis. 34, 345-351. doi: 10.2307/1591417

Reed, L. J., and Muench, H. (1938). A simple method of estimation of fifty percent end points. Am. J. Hygiene 27, 493-497.

Roan, D. T., Thanh Huong, D. T., Kim Xuyen, L. T., Khue, N. T., Tien, V. T., and Hoa, L. T. (2015). Genotyping of duck hepatitis virus type 3 isolated in Vietnam during 2009-2013. Tap. Chi. Sinh. Hoc. 37, 274-278. doi: 10.3855/jidc. 7239

Roh, J. H., and Kang, M. (2018). Live attenuated duck hepatitis virus vaccine in breeder ducks: Protective efficacy and kinetics of maternally derived antibodies. Vet. Microbiol. 219, 107-112. doi: 10.1016/j.vetmic.2018. 04.021

Soliman, M., Alfajaro, M. M., Lee, M. H., Jeong, Y. J., Kim, D. S., Son, K. Y., et al. (2015). The prevalence of duck hepatitis A virus types 1 and 3 on Korean duck farms. Arch. Virol. 160, 493-498. doi: 10.1007/s00705-0142264-3

Sun, Y., Yang, C., Li, J., Li, L., Cao, M., Li, Q., et al. (2017). Construction of a recombinant duck enteritis virus vaccine expressing hemagglutinin of H9N2 avian influenza virus and evaluation of its efficacy in ducks. Arch. Virol. 162, 171-179. doi: 10.1007/s00705-016-3077-3

Szymczak-Workman, A. L., Vignali, K. M., and Vignali, D. A. A. (2012). Design and construction of $2 \mathrm{~A}$ peptide-linked multicistronic vectors. Cold Spring Harb. Protoc. 2012, 199-204. doi: 10.1101/pdb.ip06 7876

Tseng, C. H., and Tsai, H. J. (2007). Molecular characterization of a new serotype of duck hepatitis virus. Virus Res. 126, 19-31. doi: 10.1016/j.virusres.2007. 01.012

Vannucc, L., Lai, M., Chiuppesi, F., Ceccherini-Nelli, L., and Pistello, M. (2013). Viral vectors: a look back and ahead on gene transfer technology. New Microbiol. 36, 1-22.

Wang, A., Gu, L., Wu, S., and Zhu, S. (2018). Duck hepatitis A virus structural proteins expressed in insect cells self-assemble into virus-like particles with strong immunogenicity in ducklings. Vet. Microbiol. 215, 23-28. doi: 10.1016/j. vetmic.2017.12.020

Wang, L., Pan, M., Fu, Y., and Zhang, D. (2008). Classification of duck hepatitis virus into three genotypes based on molecular evolutionary analysis. Virus Genes 37, 52-59. doi: 10.1007/s11262-0080233-1 
Wen, X., Zhu, D., Cheng, A., Wang, M., Chen, S., Jia, R., et al. (2018). Molecular epidemiology of duck hepatitis a virus types 1 and 3 in China, 2010-2015. Transbound Emerg. Dis. 65, 10-15. doi: 10.1111/tbed.12741

Yang, C., Li, J., Li, Q., Li, H., Xia, Y., Guo, X., et al. (2013). Complete genome sequence of an attenuated duck enteritis virus obtained by in vitro serial passage. Genome Announc. 1, e613-e685. doi: 10.1128/genomeA.00 $685-13$

Yang, C., Li, Q., Li, J., Zhang, G., Li, H., Xia, Y., et al. (2014). Comparative genomic sequence analysis between a standard challenge strain and a vaccine strain of duck enteritis virus in China. Virus Genes 48, 296-303. doi: 10.1007/s11262013-1009-9

Yugo, D. M., Hauck, R., Shivaprasad, H. L., and Meng, X. J. (2016). Hepatitis virus infections in poultry. Avian Dis. 60, 576-588. doi: 10.1637/11229-070515Review.1

Zou, Z., Ma, J., Huang, K., Chen, H., Liu, Z., and Jin, M. (2016). Live attenuated vaccine based on duck enteritis virus against duck hepatitis a virus types 1 and 3. Front. Microbiol. 7:1613. doi: 10.3389/fmicb.2016.01613
Conflict of Interest: The authors declare that the research was conducted in the absence of any commercial or financial relationships that could be construed as a potential conflict of interest.

Publisher's Note: All claims expressed in this article are solely those of the authors and do not necessarily represent those of their affiliated organizations, or those of the publisher, the editors and the reviewers. Any product that may be evaluated in this article, or claim that may be made by its manufacturer, is not guaranteed or endorsed by the publisher.

Copyright (๑) 2021 Yang, Liu, Li, Liu, Gao, Cui, Zhang, Liu, Qi, Pan, Liu, Wang, Gao and $L i$. This is an open-access article distributed under the terms of the Creative Commons Attribution License (CC BY). The use, distribution or reproduction in other forums is permitted, provided the original author(s) and the copyright owner(s) are credited and that the original publication in this journal is cited, in accordance with accepted academic practice. No use, distribution or reproduction is permitted which does not comply with these terms. 